\title{
Analysis of homozygosity disequilibrium using whole-genome sequencing data
}

\author{
Hsin-Chou Yang*, Han-Wei Li \\ From Genetic Analysis Workshop 18 \\ Stevenson, WA, USA. 13-17 October 2012
}

\begin{abstract}
Homozygosity disequilibrium (HD), a nonrandom sizable run of homozygosity in the genome, may be related to the evolution of populations and may also confer susceptibility to disease. No studies have investigated HD using whole genome sequencing (WGS) analysis. In this study, we used an enhanced version of Loss-Of-Heterozygosity Analysis Suite (LOHAS) software to investigate HD through analysis of real and simulated WGS data sets provided by Genetic Analysis Workshop 18. Using a local polynomial model, we derived whole-genome profiles of homozygosity intensities for 959 individuals and characterized the patterns of HD. Generalized estimating equation analysis for 855 related samples was performed to examine the association between patterns of HD and 3 phenotypes of interest, namely diastolic blood pressure, systolic blood pressure, and hypertension status, with covariate adjustments for age and gender. We found that $4.48 \%$ of individuals in this study carried sizable runs of homozygosity (ROHs). Distributions of the length of $\mathrm{ROH}$ s were derived and revealed a familial aggregation of $\mathrm{HD}$. Genome-wide homozygosity association analysis identified 5 and 3 ROHs associated with diastolic blood pressure and hypertension, respectively. These regions contain genes associated with calcium channels (CACNA1S), renin catalysis (REN), blood groups (ABO), apolipoprotein (APOA5), and cardiovascular diseases (RASGRP1). Simulation studies showed that our homozygosity association tests controlled type 1 error well and had a promising power. This study provides a useful analysis tool for studying HD and allows us to gain a deeper understanding of HD in the human genome.
\end{abstract}

\section{Background}

Homozygosity disequilibrium (HD) describes a phenomenon in which a nonrandom pattern is observed for a sizable run of homozygosity ( $\mathrm{ROH}$ ) in the human genome, where $\mathrm{ROH}$ indicates a contiguous set of homozygous genotypes in an intact genomic region or allows to be interrupted by a small proportion of heterozygous genotypes arising from genotyping errors, missing genotypes, or mutations [1]. HD can result from autozygosity [2], natural selection [3], and chromosomal aberrations [4]. Previous studies suggested that HD may confer susceptibility to neurodevelopment-related disorders [5,6] and autoimmune diseases [1,7]. No studies have investigated HD with whole genome sequencing (WGS) analysis. This study analyzed a real human WGS data set and simulated

\footnotetext{
* Correspondence: hsinchou@stat.sinica.edu.tw

Institute of Statistical Science, Academia Sinica, Nankang 115, Taipei, Taiwan
}

data sets provided by Genetic Analysis Workshop 18 (GAW18) with four major aims. The first aim was to develop statistical methods and analysis tools to examine HD in WGS data. The second aim was to characterize patterns of HD in the human genome. The third aim was to identify regions of HD associated with diastolic blood pressure (DBP), systolic blood pressure (SBP), and hypertension status. The final aim of this study was to evaluate the performance of the proposed genome-wide homozygosity association analysis approach on the simulated data set. This study constitutes a useful resource for examining $\mathrm{HD}$ and provides insight into the potential roles of $\mathrm{HD}$ in population genetics and medical genetics.

\section{Methods \\ Materials}

GAW18 provided a combined imputation data set derived from deep sequencing data for the whole genomes of 464 
individuals and genome-wide association genotype data for 495 individuals. All 959 individuals were from 20 large independent pedigrees enrolled in the T2D-GENES (Type 2 Diabetes Genetic Exploration by Next-generation sequencing in Ethnic Samples) Project 2. Full information about blood pressures (DBP and SBP) and covariates (age and gender) was also available for 855 of these individuals. In this study, an individual was considered hypertensive if he or she had ever taken antihypertensive medication or his or her DBP was greater than $90 \mathrm{~mm} \mathrm{Hg}$ or SBP was greater than $140 \mathrm{~mm} \mathrm{Hg}$ at the most recent examination. The genome of each individual was sequenced by Complete Genomics with an average depth of coverage of 60x. Multiple quality control procedures were carried out to filter out single-nucleotide variants (SNVs) with poor performance in allele balance, strand bias, fraction of bases with low quality, and Mendelian errors by GAW18. The WGS data set of 464 individuals contained 24 million of SNVs that passed quality filters, and more than $51 \%$ of them were rare variants (RVs). The combined data set of 959 individuals contained 8,348,663 single-nucleotide polymorphisms (SNPs) and 5,573,886 RVs for oddnumbered autosomes. In addition, GAW18 provided 200 simulation data sets of quantitative trait Q1. Q1 was generated from a normal distribution and was independent of genetic variants in this study.

\section{Statistical methods}

For this study, we developed an enhanced version of the Loss-Of-Heterozygosity Analysis Suite (LOHAS) software [8]. LOHAS was originally developed for detecting loss of heterozygosity in cancer research and identifying long contiguous stretches of homozygosity in population genetics studies using SNP genotype data. LOHAS provides a two-step procedure for homozygosity association studies. First, LOHAS constructs sliding windows on a chromosome using a nearest neighbor method and estimates homozygosity intensity in each window for each individual using a local polynomial model. In the model, the homozygote-heterozygote status was regressed by the physical positions of SNVs to calculate the homozygosity intensity. Homozygosity intensities are values between 0 and 1 . Second, LOHAS performs a linearrank association test to identify runs of homozygosity (ROHs) with differential homozygosity intensities between the study groups.

We extended LOHAS to handle large numbers of SNPs and RVs in the WGS data by introducing the following adapted coding system of homozygote-heterozygote status. Major and minor alleles at a SNV were $\operatorname{denoted} A$ and $a$, respectively. SNVs were classified as SNPs if their minor allele frequency (MAF) was higher than 0.05 or as RVs if lower than 0.05, where MAF was calculated across the whole population. For the SNP analysis, the coding system was the same as that used in the original LOHAS software. Homozygotes ( $a a$ and $A A$ ) were coded as 1 and heterozygotes $(A a)$ as 0 . For the RV analysis, rare homozygotes $(a a)$ and heterozygotes $(A a)$ were coded as 1 and 0 , respectively. Because common homozygotes $(A A)$ of RVs are less informative and therefore could dilute the assessment of other informative homozygotes when defining $\mathrm{ROH}$, they were analyzed separately. Homozygosity intensity of a window anchored at a common homozygote of a RV was estimated by inputting the physical position of the anchor into the fitted local polynomial model, which was built based on genotypes except for common homozygotes of RVs. We also expanded LOHAS software by adding model-based methods for studying a dichotomous or quantitative trait with or without an adjustment for covariates. A new analysis module of generalized estimating equation (GEE) was included for analysis of related individuals, and a new analysis module of linear regression model was included for analysis of unrelated individuals.

To estimate whole-genome homozygosity intensities of all 959 individuals, a window size, $5 \%$ of SNVs on a chromosome, was considered. Each window contained 11,968 to 65,465 SNVs. The GEE was used to analyze 855 related samples with WGS data, blood pressures, hypertension status, and covariates. Homozygosity intensities were modeled as a continuous response to correlate with DBP values, SBP values, and hypertension status, with concomitant adjustment for age and gender.

We also evaluated type 1 error and test power of the homozygosity association tests using simulation data. Type 1 error was analyzed by examining the association between homozygosity intensities and Q1 (with covariate adjustments for age and gender) using the GEE analysis. We calculated the proportion of times that the null hypothesis was rejected over 200 simulations at each window and then calculated the average type 1 error over all windows. Test power was analyzed by choosing 3 regions on chromosome 21 containing 200 SNVs for each with varying proportions of RVs. The 3 regions contained $51.5 \%, 66.5 \%$, and $80.0 \%$ RVs representing the 10th, 50th, and 90th percentiles of proportions of RVs on chromosome 21, respectively. The 3 regions were designated as Q1-associated ROHs by a logistic regression model as follows:

$$
\operatorname{Logit}(p)=b_{0}+b_{1} \cdot \mathrm{Q} 1,
$$

where $p$ is the probability that all genotypes in the region were replaced by homozygotes and $b_{0}$ and $b_{1}$ are the regression coefficients. The test power was calculated as the proportion of times that the null hypothesis was rejected over 200 simulations at a Q1-associated $\mathrm{ROH}$. 


\section{Results}

Our analyses were performed without knowledge of the underlying simulation model. Whole-genome profiles of homozygosity intensities for 959 individuals were derived from the GAW18 data set. Approximately 4.48\% of individuals (43/959) carried sizable ROHs with homozygosity intensities greater than 0.9 and run lengths greater than $5 \mathrm{Mb}$. The minimum, 25th, 50th, and 75th percentiles and maximum of $\mathrm{ROH}$ lengths were $5.07,5.66$, $7.40,13.61$, and $44.60 \mathrm{Mb}$, respectively. The minimum, 25th, 50th, and 75th percentiles and maximum of total lengths of ROHs carried by an individual were 5.07, 5.64, $7.54,14.81$, and $105.85 \mathrm{Mb}$, respectively. Figure 1 shows an example of a male subject (T2DG0300128) who carries multiple ROHs on chromosomes $1,5,9,11,13,15$, and 19. We also observed a familial aggregation of ROHs. For example, four individuals (T2DG2701096, T2DG2701097, T2DG2701098, and T2DG2701099) in pedigree 27 carried the identical ROHs on chromosomes 13 and 21.

We carried out whole-genome homozygosity tests to identify DBP-, SBP-, or hypertension-associated ROHs. GEE analysis of 855 related individuals identified several DBP- or hypertension-associated ROHs after controlling for false discovery rate (Figure 2). DBP-associated $\mathrm{ROHs}$ were located in the regions ranged from 198.4 to 210.0
$\mathrm{Mb}$ on chromosome 1 (adjusted $p$-value $=0.0396$ ), from 31.9 to $38.1 \mathrm{Mb}$ on chromosome 9 (adjusted $p$-value = 0.0396 ), from 41.4 to $48.5 \mathrm{Mb}$ (adjusted $p$-value $=0.0396$ ) and from 97.7 to $103.3 \mathrm{Mb}$ (adjusted $p$-value $=0.0396$ ) on chromosome 11, and from 108.0 to $112.1 \mathrm{Mb}$ on chromosome 13 (adjusted $p$-value $=0.0396$ ). Hypertensionassociated ROHs were located in the regions ranged from 132.7 to $137.9 \mathrm{Mb}$ on chromosome 9 (adjusted $p$-value $=$ 0.0264 ), from 109.5 to $116.1 \mathrm{Mb}$ on chromosome 11 (adjusted $p$-value $=0.0050$ ), and from 33.7 to $37.5 \mathrm{Mb}$ on chromosome 15 (adjusted $p$-value $=0.0050$ ). No SBPassociated $\mathrm{ROHs}$ were found.

We evaluated type 1 error of the homozygosity association tests by analyzing 200 simulation data sets of quantitative trait Q1. For a GEE association analysis of the 855 related individuals, the mean of type 1 errors was 0.03 . We also evaluated the test power of our homozygosity association tests by analyzing the 200 simulation data sets with 3 Q1-associated ROHs created under the parameters that $b_{0}=-20$ and $b_{1}=0.5$ in our simulation model. For the GEE analysis of 855 related individuals, the power for the 3 Q1-associated ROHs containing $51.5 \%, 66.5 \%$, and $80.0 \%$ RVs was $1.00,1.00$, and 1.00 , respectively. The power was reduced to $0.910,0.975$, and 0.945 when $b_{0}=-20$ was changed to $b_{0}=-10$.

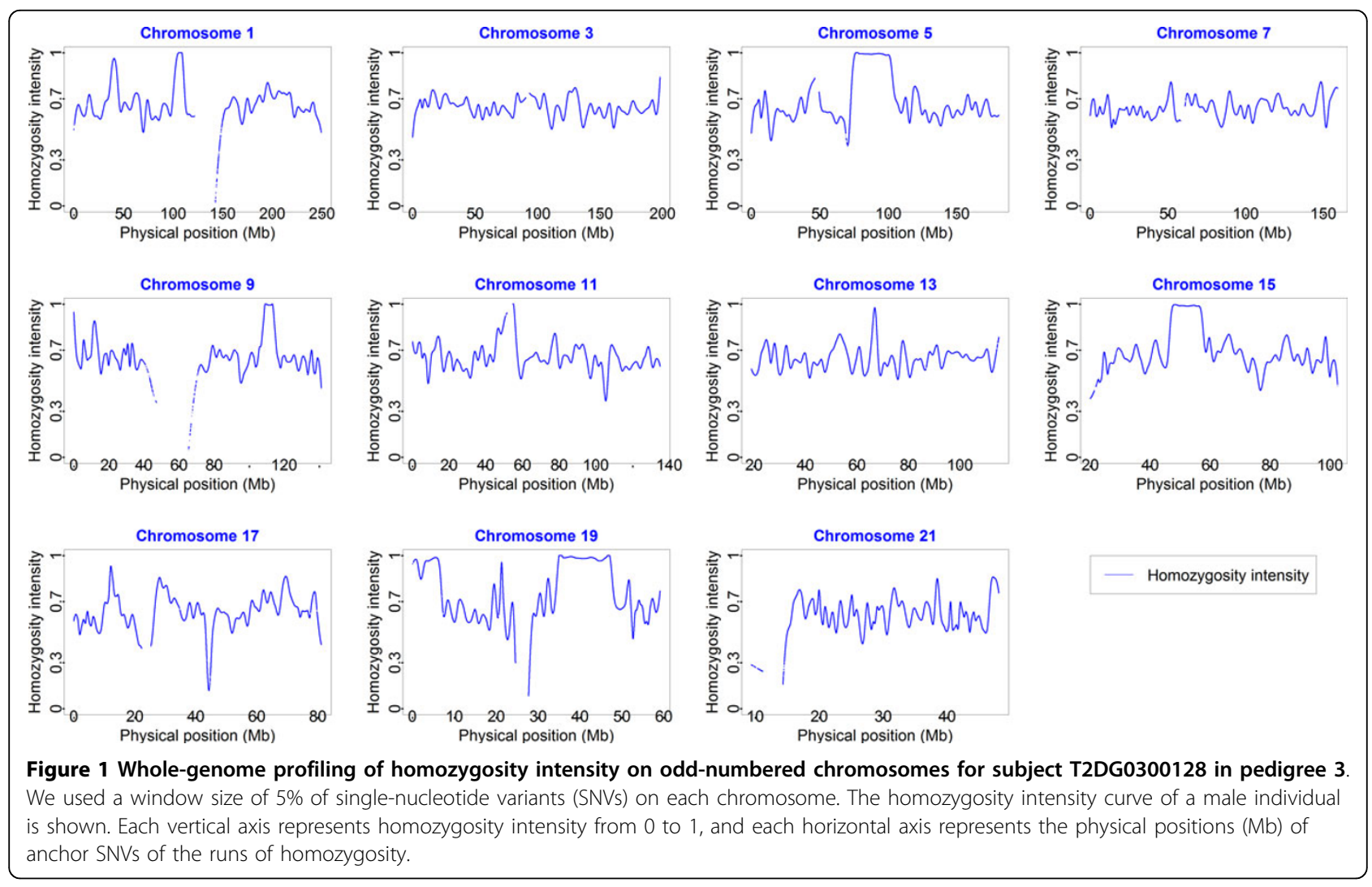




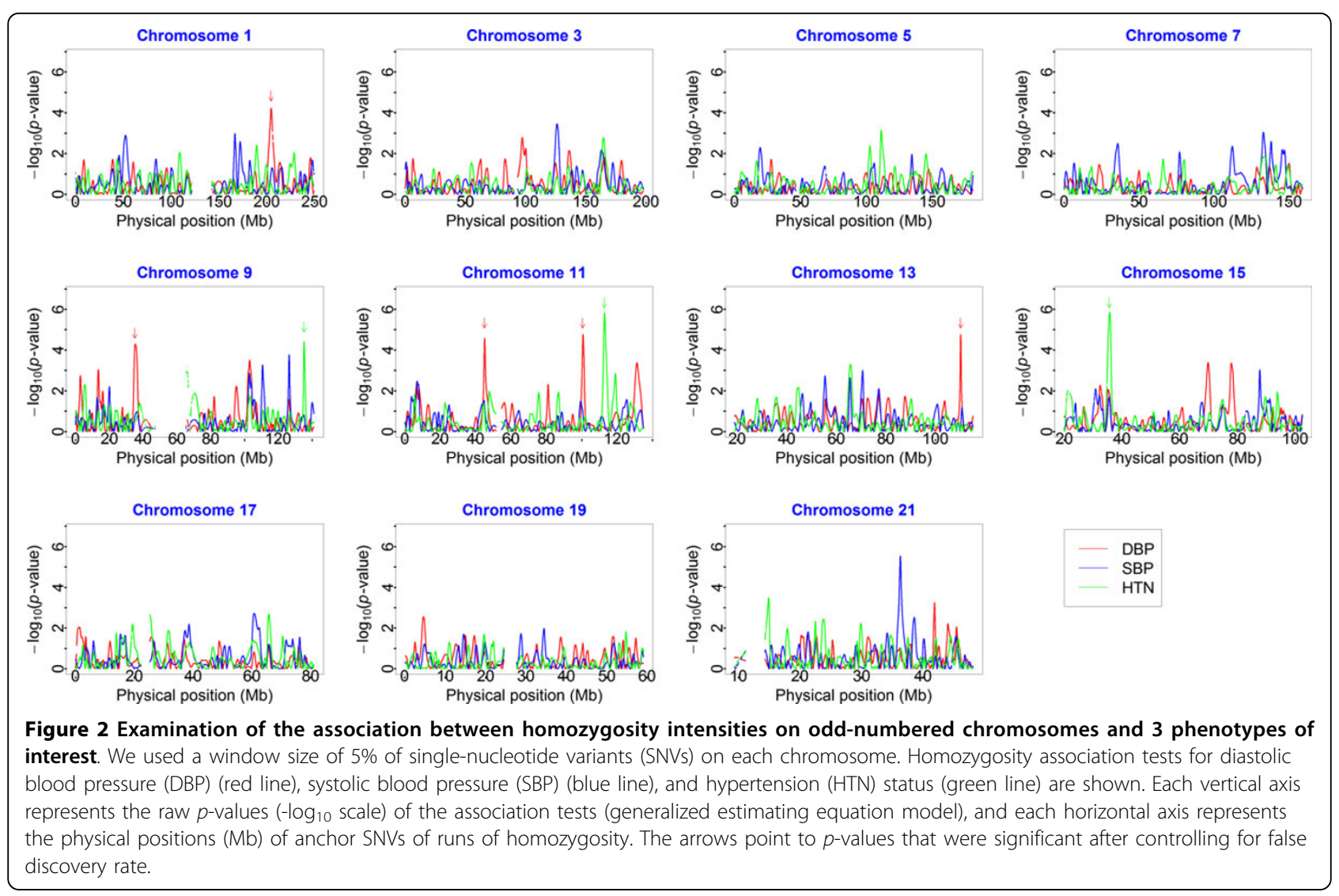

\section{Discussion and conclusions}

This study has made several contributions to WGS data analysis of HD in the human genome. First, we adapted LOHAS software to enable more complete analysis of WGS data for HD. Two new homozygosity association analysis modules, a regression-based analysis for unrelated individuals and a GEE-based analysis for related individuals, have been incorporated into LOHAS. Software, examples, and user guide are now available free of charge at http://www.stat.sinica.edu.tw/hsinchou/genetics/loh/ LOHAS.htm.

Second, we characterized the patterns of HD by analyzing a large WGS data set of 959 individuals from 20 pedigrees. The distribution of lengths for sizable $\mathrm{ROHs}$ and a distribution of total run length for one individual were obtained. The larger individual total run lengths of an individual may suggest autozygosity owing to inbreeding, consanguineous marriage, or a recent common ancestor. This approach may also be useful in identifying specific types of chromosomal aberrations such as uniparental disomy, hemizygous deletion, and loss of heterozygosity in cancer studies. In addition, we found that patterns of HD differ among individuals. We also observed familial aggregation, suggesting a genetic component of HD. A sensitivity analysis, which considered 3 thresholds of
MAF for defining RVs (MAF $=0.04,0.05$, and 0.06), showed that the results were robust to a small change in the threshold of MAF for defining RVs. These findings suggest potential applications of these methods in genetic studies.

Third, our GEE-based homozygosity association tests identified 5 DBP-associated ROHs and 3 hypertensionassociated ROHs. Genes in these ROHs include some that are associated with calcium channels, renin catalysis, insulin-like growth factor (IGF), blood groups, apolipoprotein, cardiovascular diseases, and rat sarcoma. Specifically, within the DBP-associated ROHs, two genes on chromosome 1q32 encode proteins that inactivate calcium channels in skeletal muscle cells (CACNA1S) and play a role in renin catalysis $(R E N)$, both of which are important for the regulation of blood pressure. IGFBPL1 on 9p13.1 encodes IGF binding protein. Within the hypertension-associated ROHs, $A B O$ on $9 \mathrm{q} 34.2$ is associated with the ABO blood group system. APOA1, APOA4, APOA5, and APOC3 on 11q23-q24 encode apolipoproteins and have been associated with cardiovascular diseases. RASGRP1 on $15 \mathrm{q} 14$ encodes a protein that functions as a Ras activator as well as a switch that is regulated by calcium and diacylglycerol, and guanosine triphosphate-guanosine diphosphate exchange. However, these results are still preliminary, and 
the findings should be further investigated in future studies.

We also examined homozygosity associations of 142 samples from unrelated individuals ( 85 hypertensive cases and 57 normotensive control participants) using a linear regression analysis. However, probably owing to the relatively small number of samples, none of the ROHs in this analysis was found to be associated with DBP, SBP, or hypertension after controlling for false discovery rate.

In summary, the enhanced LOHAS developed in this study represents a useful tool for studying HD under different ascertainment schemes (unrelated and related individuals), phenotypes of interest (dichotomous disease status and quantitative traits), and experimental platforms (SNP microarrays and next-generation sequencing experiments). Performance of the improved LOHAS homozygosity association tests was evaluated by simulation studies, and the results suggest that the methods are reliable with a high test power and wellcontrolled type 1 error. Additional simulation studies and real data analyses will further elucidate the limitations of our methods and helps us further understand HD in the human genome.

\section{Competing interests}

The authors declare that they have no competing interests.

\section{Authors' contributions}

HCY conceived of the study, developed statistical methods, and prepared the manuscript. HWL developed analysis software and analyzed the data with HCY. Both authors read and approved the final manuscript.

\begin{abstract}
Acknowledgements
We are grateful to GAW18 for providing the genomic data set. We thank Dr. Andrew Paterson for constructive suggestions in the discussion at the group meeting at GAW18. We thank two anonymous reviewers and the editor for constructive suggestions for this paper. The Workshop was supported by National Institutes of Health (NIH) USA grants. This work was partially supported by grants from the National Science Council of Taiwan (NSC 1002314-B-001-005-MY3) and the Career Development Award of Academia Sinica (AS-100-CDA-M03). The GAW18 WGS data were provided by the T2DGENES Consortium, which is supported by NIH grants U01 DK085524, U01 DK085584, U01 DK085501, U01 DK085526, and U01 DK085545. The other genetic and phenotypic data for GAW18 were provided by the San Antonio Family Heart Study and San Antonio Family Diabetes/Gallbladder Study, which are supported by NIH grants P01 HL045222, R01 DK047482, and R01 DK053889. The GAW is supported by NIH grant R01 GM031575. This article has been published as part of BMC Proceedings Volume 8 Supplement 1, 2014: Genetic Analysis Workshop 18. The full contents of the supplement are available online at http://www.biomedcentral.com/bmcproc/ supplements/8/S1. Publication charges for this supplement were funded by the Texas Biomedical Research Institute.
\end{abstract}

Published: 17 June 2014

\section{References}

1. Yang HC, Chang LC, Liang YJ, Lin CH, Wang PL: A genome-wide homozygosity association study identifies runs of homozygosity associated with rheumatoid arthritis in the human major histocompatibility complex. PLOS ONE 2012, 7:e34840.

2. Gibson J, Morton NE, Collins A: Extended tracts of homozygosity in outbred human populations. Hum Mol Genet 2006, 15:789-795.
3. Sabeti PC, Varilly P, Fry B, Lohmueller J, Hostetter E, Cotsapas C, Xie XH, Byrne EH, McCarroll SA, Gaudet R, et al: Genome-wide detection and characterization of positive selection in human populations. Nature 2007, 449:913-918.

4. Cavenee WK, Dryja TP, Phillips RA, Benedict WF, Godbout R, Gallie BL, Murphree AL, Strong LC, White RL: Expression of recessive alleles by chromosomal mechanisms in retinoblastoma. Nature 1983, 305:779-784.

5. Nalls MA, Guerreiro RJ, Simon-Sanchez J, Bras JT, Traynor BJ, Gibbs JR, Launer L, Hardy J, Singleton AB: Extended tracts of homozygosity identify novel candidate genes associated with late-onset Alzheimer's disease. Neurogenetics 2009, 10:183-190.

6. Lencz T, Lambert C, DeRosse P, Burdick KE, Morgan TV, Kane JM, Kucherlapati R, Malhotra AK: Runs of homozygosity reveal highly penetrant recessive loci in schizophrenia. Proc Natl Acad Sci U S A 2007, 104:19942-19947.

7. Baschal EE, Aly TA, Jasinski JM, Steck AK, Noble JA, Erlich HA, Eisenbarth GS: Defining multiple common "completely" conserved major histocompatibility complex SNP haplotypes. Clin Immunol 2009, 132:203-214.

8. Yang HC, Chang LC, Huggins RM, Chen CH, Mullighan CG: LOHAS: loss-ofheterozygosity analysis suite. Genet Epidemiol 2011, 35:247-260.

doi:10.1186/1753-6561-8-S1-S15

Cite this article as: Yang and Li: Analysis of homozygosity disequilibrium using whole-genome sequencing data. BMC Proceedings 2014 8(Suppl 1):S15.

\section{Submit your next manuscript to BioMed Central and take full advantage of:}

- Convenient online submission

- Thorough peer review

- No space constraints or color figure charges

- Immediate publication on acceptance

- Inclusion in PubMed, CAS, Scopus and Google Scholar

- Research which is freely available for redistribution 